\title{
A Qualitative Study of the Communication Process for Medical Acupuncture in Family Medicine
}

\author{
Christy J.W. Ledford, PhD; Carla L. Fisher, PhD; Paul Crawford, MD
}

\begin{abstract}
BACKGROUND AND OBJECTIVES: As evidence establishes the efficacy of medical acupuncture, more family physicians and family medicine residents may receive medical acupuncture training and need to know how to effectively communicate about the treatment option with patients. By identifying how physicians talk about acupuncture treatment with their patients, we aimed to develop a model for physician training that could enhance their ability to integrate and practice medical acupuncture in conventional clinical settings.
\end{abstract}

METHODS: To capture the communication process that family physicians engage in when integrating acupuncture treatment into a clinical environment, we sought both physicians' and patients' perspectives. We conducted interviews with 17 family physicians and 15 patients in a US family medicine clinic that has integrated medical acupuncture into its practice. Audio recordings were transcribed and analyzed by two members of the study team in ATLAS.ti, using the constant comparative method.

RESULTS: Integrating acupuncture into family medicine entailed a three-phase communication process: (1) introduce acupuncture, (2) explain the medical process, and (3) evaluate treatment outcomes.

CONCLUSIONS: The emerging three-phase process of communicating acupuncture described here provides an initial model for teaching communication in the context of medical acupuncture. Given the exploratory nature of this initial study and the rarity of acupuncture treatment integrated into family medical settings, this is a first step in building knowledge in this realm of practice. Future research is needed to better understand the experience of patients who do not report notable results of acupuncture and to extend this study into other family medicine settings.

(Fam Med. 2018;50(5):353-8.)

doi: 10.22454/FamMed.2018.662450

$\mathbf{M}$ edical acupuncture can provide successful treatment for patients coping with chronic and acute mental and physical ailments. ${ }^{1-4}$ Defined as the therapeutic insertion of solid needles in various combinations and patterns, ${ }^{5}$ medical acupuncture encompasses
French energetic acupuncture, trigger point acupuncture, Chinese scalp acupuncture, comprehensive auricular acupuncture, electroacupuncture, and Worlsey five-element acupuncture. ${ }^{6}$ As evidence establishes the efficacy of medical acupuncture, ,,7-11 more family physicians and family medicine residents will likely receive medical acupuncture training and need to know how to effectively communicate about the treatment option with patients.

The practice of medical acupuncture is a unique context for studying clinical communication between patient and physician. In the context of acupuncture, research on the therapeutic benefits of clinician-patient communication has hypothesized effects of communication on patient outcomes. For example, an augmented communication style such as expressing empathy and active listening and positive expectations for success has been associated with patients perceiving lower symptom severity of irritable bowel syndrome. ${ }^{12}$ When acupuncturists expressed hope and optimism that treatment would reduce pain and improve function, patients reported better jointspecific pain control compared to patients whose acupuncturists expressed uncertainty and a "wait and see" approach about whether treatment would work. ${ }^{13}$ In one study with nonphysician acupuncturists,

From the Department of Family Medicine, Uniformed Services University of the Health Sciences, Bethesda, MD (Dr Ledford); STEM Translational Communication Center, University of Florida Cancer Center, Gainesville, FL (Dr Fisher); and Nellis Family Medicine Residency Program, Mike O'Calaghan Military Medical Center, Nellis Air Force Base, NV (Dr Crawford). 
the providers' communication of optimism about treatment effectiveness was associated with pain and function outcomes through its effect on satisfaction during treatment. ${ }^{14}$ These studies demonstrate how physician communication about medical acupuncture can enhance patient outcomes.

Although we are increasingly teaching physicians how to provide acupuncture ${ }^{15}$ we have given less attention to teaching physicians how to talk to patients about acupuncture. To capture family physicians' communication process when integrating acupuncture into a clinical environment in which patients may be less familiar with this form of treatment, we sought both physicians' and patients' perspectives. In doing so, we can more comprehensively identify a communication process for integrating this form of care into conventional medicine. Given that research shows that talking about treatment efficacy enhances patient participation, ${ }^{16-18}$ we paid close attention to both patients' and physicians' perceptions of what helped facilitate acupuncture engagement and perceptions of treatment efficacy or outcomes. By identifying how physicians talk about acupuncture treatment with their patients, we aim to develop a model for physician training that could enhance their ability to integrate and practice medical acupuncture in conventional clinical settings.

\section{Methods}

Following institutional review board approval, research coordinators recruited 15 family medicine physicians and 17 patients $(\mathrm{N}=32)$ from a family medicine department at a US acute/emergency medical center with a patient population of 50,000. Physician participants had completed a 300-hour medical acupuncture course, at sites outside of our health system in Arizona and Maryland and also at our site in Nevada. Physician and patient groups included participants who had practiced/received acupuncture at our clinical site and in other health systems. See Table 1 for demographics.

On-site coordinators obtained consent and demographics. Two authors conducted individual semistructured in-depth interviews lasting about 1 hour each during three separate site visits. Those interviews were then professionally transcribed with a coding system to maintain confidentiality, which resulted in 812 transcribed pages. Each site visit lasted 2 to 3 days. Interviews occurred in the clinic in a private room to maintain privacy, enhance access, and reduce participation burden.

The findings reported for the posited inquiry are one segment of a larger study. Participants were asked about the nature of their interactions as they pertained to engaging in acupuncture treatment. The larger study focused on what physicians and patients discussed as it

Table 1: Participant Demographics

\begin{tabular}{|l|l|}
\hline \multicolumn{2}{|c|}{ Physician Participants (n=15) } \\
\hline Gender & $3(20 \%)$ \\
\hline Female & $12(80 \%)$ \\
\hline Male & Mean 3.73 (SD 1.94) \\
\hline Years practicing medicine & Mean 2.00 (SD 1.31) \\
\hline Years practicing medical acupuncture & \multicolumn{1}{|c|}{ Patient Participants (n=17) } \\
\hline \multicolumn{2}{|c|}{} \\
\hline Gender & $10(58.8 \%)$ \\
\hline Female & $7(41.2 \%)$ \\
\hline Male & Mean 52.18 (SD 13.93) \\
\hline Age in years & $4(23.5 \%)$ \\
\hline Number of acupuncture treatments received & $9(52.9 \%)$ \\
\hline Low utilizer (up to 5 treatments) & $4(23.5 \%)$ \\
\hline Medium utilizer (6 to 20 treatmnets) & $8(47.1 \%)$ \\
\hline High utilizer (more than 20 treatments) & $5(29.4 \%)$ \\
\hline Primary reason for receiving acupuncture treatment* & $4(23.5 \%)$ \\
\hline Chronic pain** &
\end{tabular}

*List indicates primary reason for receiving acupuncture. Multiple patients reported receiving acupuncture for more than one reason.

**Includes persistent migraine. 
pertained to engaging in acupuncture treatment, with a heavy focus on what communicative approaches enhanced patients' willingness to engage in and maintain acupuncture as well as perceived health outcomes. Beginning with the first site visit, researchers kept memos to develop themes relevant to inquiries posed in the larger study, meeting to discuss findings after interviews as well as at the end of each data collection day. These analytical meetings were conducted so that reports could be compared to further develop patterns, enhance reflexivity, and attain thematic saturation. During this data collection and concurrent analysis it was noted that, without any probing, patients and physicians were describing a process of communication that illustrated how acupuncture was integrated into the family medicine setting. This was integrated into analysis and ongoing data collection to develop a richer description of a communication process that physicians could use when integrating acupuncture into family medicine settings.

Thematic analysis was conducted using the constant comparative method, ${ }^{19}$ including memo keeping to maintain reflexivity and reach thematic saturation, using these analytical steps: (1) identifying concepts/ assigning codes, (2) establishing categories, and (3) refining themes/characteristics with rich descriptions. ${ }^{20}$ The large data set was managed using ATLAS.ti. Analysis was separated by each group (patient and physician) and compared. Similar findings were collapsed to develop an illustration of the entire communication process with any distinctions noted in perception for descriptive value (eg, the first phase was more physician-led as opposed to the second phase which was more patientdriven). Saturation of themes was met when at least half of participants in a group reported that phenomenon. Patterns or categories were refined to identify dimensions (features of the communication process) to further define categories and ensure rich description.

\section{Results}

Integrating acupuncture into family medicine entailed a three-phase communication process: (1) introduce acupuncture, (2) explain the medical process, and (3) evaluate treatment outcomes (Figure 1). The sequential nature of the model emerged from the data from both descriptions of current practice as well as patient recommendations for preferred practice. Excerpts illustrate the key communication features of each phase and provide exemplars that can be used to not only create a communication process for acupuncture integration, but also for teaching and testing the efficacy of this communication process.

\section{Introduce Acupuncture}

Physicians would first introduce acupuncture as a treatment option. This involved three physician-led communication features: (1) initiate discussion, (2) use a collaborative communication style, and (3) mention treatment efficacy. One physician described how he enacted this phase and incorporated each communication feature in his introduction:

I say [to my patients], "Listen.

Sometimes [back pain] is a tricky thing to treat. These are our options. You can do physical therapy. We can do medications, which isn't a good thing to have long-term. But also something that we've been starting to get trained [in] is acupuncture. And a lot of people have been getting battlefield acupuncture and ear acupuncture... We've been learning a lot more of the full body acupuncture and been having really great results with people and that could be something that could be really beneficial..." Most of the time [patients are] really open to that... I say [to them] "So what are your thoughts on those options?" or "What do you think would benefit you?" (Dr3)

This phase of the communication process received the most attention and time in the clinical interaction and was also more dominated by the physician talking. Physicians reported that this was necessary

Figure 1: Communication Process Through Medical Acupuncture

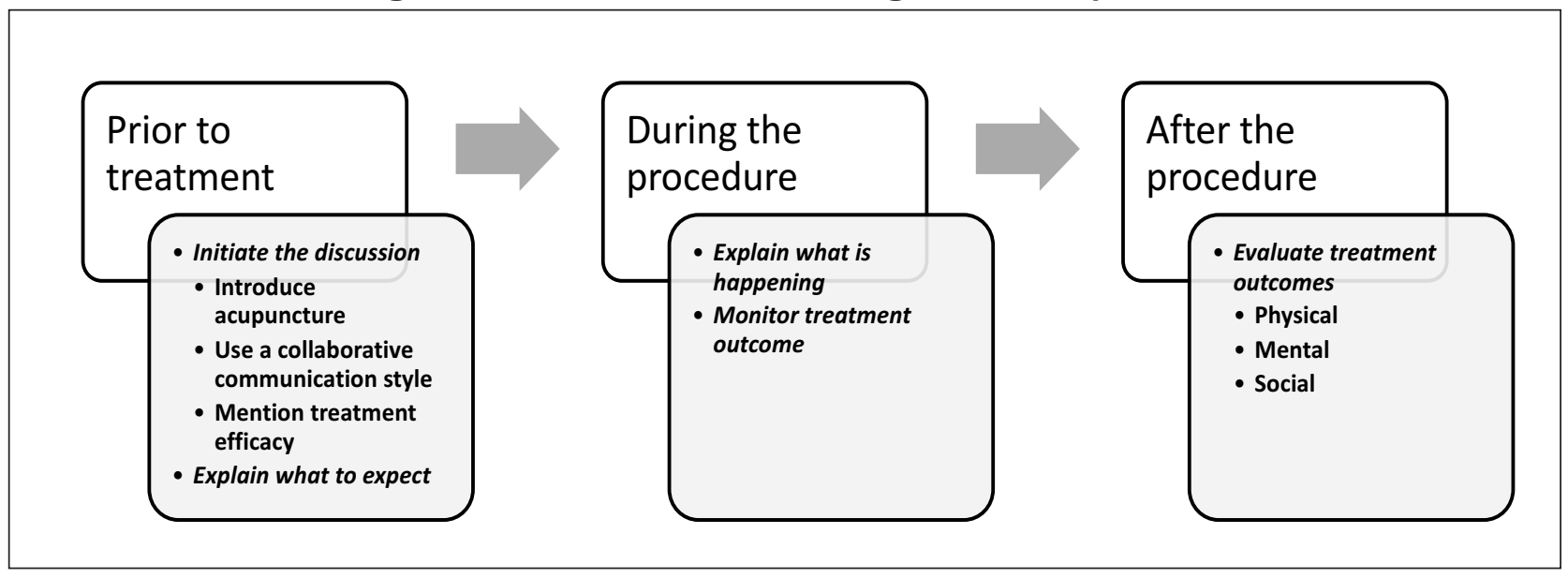


to address an unfamiliar treatment that could be misunderstood. Likewise, patients described this phase as critical for addressing issues pertinent to treatment decisions, which could warrant more extensive conversations.

\section{Explain the Medical Process}

Physicians would then explain the medical process. This phase involved two physician-led communication features: (1) explain what to expect, and (2) what happens when acupuncture is administered. This explanation prepared and educated patients and also provided an opportunity to address needle aversion, a common issue for patients. One patient described the importance of this phase and associated communication features and also noted an explanatory tactic regarding needles that enhanced his comfort:

The more [physicians] explain what [acupuncture] is, how they're treating [the health issue] and why, I think it would make the patients more comfortable because a lot of people don't like the thing about all these needles in them. One doctor didn't distract me, I felt the needle go in... It was funny but it really works-that distraction actually works... Communication wise would be explain what you're doing and why. Don't just make a determination [such as], "We'll stick you with a needle and that's it..." I think a lot of people are hesitant because of the fact that this is new-different. (Pt12)

This phase also included a patient-driven communication feature in which physicians responded to patient cues to determine depth/ breadth of explanation (eg, whether to integrate Eastern medicine or scientific physiological explanations). Physicians also paid attention to changes in cues across treatment, as this physician illustrated:

I used to explain every little thing [to patients]. Then I realized they don't even want to hear it. Like it doesn't matter if I say nothing or say everything. So I just tell them what they're going to feel so they don't get surprised by it... A handful I would say while we're doing [acupuncture] maybe this is the second or third time and they know it works-they [ask me], "So how does this even work?" They ask questions like that. (Dr11)

\section{Evaluate Treatment Outcomes}

During the final phase physicians would evaluate treatment outcomes. This involved a physician-led (but collaborative) communication feature to longitudinally evaluate outcomes (1) during treatment, (2) after treatment, and (3) at follow-up appointments/treatments. While physicians initiated communication, patients were collaboratively involved by assessing outcomes both quantitatively (eg, rating pain on a scale of 1-10) and qualitatively (eg, describing how you felt this week). Physicians noted that evaluating outcomes was important not only for treatment decisions, but also to validate their practice of acupuncture accurately as new acupuncture practitioners.

This phase was challenging, warranting more time with both evaluation approaches to develop a dialogue, indicating a phase of the communication process that hinged on collaboration between physician and patient. One physician explained, "I feel like you really, really have to interact with the patient to figure out which [treatment] is the best" [Dr6]. That physician described probing with various questions including:

"Do you remember that scale? Where are you at now?"

"Where did your pain go after you left the clinic?"

"How long does the pain [last]—or [did] pain improve for?"

This physician also noted that assessing mental health changes was challenging and required more probing to "tease those out." This included asking about sleep patterns or relational changes with loved ones. Although this phase was recurrent, it received less attention in the process. Some patients did not recall evaluations at each time point, reported the evaluation was too narrow, or that self-monitoring was difficult. One patient helped to illustrate how evaluation of outcomes warranted a number of considerations like the patient's ability to monitor their own health, differences across patients, as well as the interlinked nature (or changing nature) of their health complaints:

Every appointment [doctors] have a paper [to evaluate my health] and it's normally only graded on the original diagnosis. For me they only really assess my back and neck pain-I mean they ask about my anxiety and insomnia but they don't write it down, well, that I see. ... They ask how your problem has been in the last week and how it is right now and how much it's hindered you in your daily activities that you list-mine is taking care of my kids and the house but for others it might be lifting or whatever. You scale from 0 to 6 or 0 to 10 ... They always ask [me] "Do you feel like it helps?" But they don't like super go into detail or have you journal... I feel like [journaling would] be kind of taxing... I think that that would stress me out and be counterproductive whereas other people might feel like that helps them be in control or whatever. [Pt7]

Although physicians and patients primarily discussed changes in health outcomes after acupuncture treatment during the evaluation phase of the communication process, they were at times incorporated into the introduction phase when efficacy was discussed. For instance, when discussing efficacy with patients, physicians could reference the success with other patients with similar ailments. Likewise, patients reported 
that they liked hearing about other patients' health improvements when being presented with acupuncture as a treatment option. Collectively, patients' and physicians' reports of outcomes centered on physical, mental, and social health improvements, which worked in tandem and were not mutually exclusive. Physical changes included a reduction or elimination of pain, opioid/narcotic use for pain, disease and ailmentrelated symptoms (eg, increased mobility, less scarring), and physiological effects associated with mental health complaints (eg, more energy, better sleep, digestion, and breathing). Mental health changes included decreased distress, enhanced mood, changes in mental health disorders (like ADHD, PTSD, anxiety, depression), and grief and trauma processing. Social health changes were also described including enhanced personal relationships at home or work as well as between physician and patient (eg, patients "opening up more" or increased trust).

\section{Discussion}

The emerging three-phase process of communicating acupuncture described here provides an initial model for teaching communication in the context of medical acupuncture. The reports help illustrate the collaborative nature of acupuncture treatment in terms of both physician-led and patient-led communication features as well as the importance of addressing critical issues (eg, what to expect, efficacy).

The first phase is not unique to this context. Previous studies examined the decision-making process to engage in innovative procedures, ${ }^{21,22}$ which would certainly describe the inclusion of acupuncture in treatment. However, the discovery of the second phase provides unique insight into physician-patient communication.

High quality patient-provider communication is associated with positive health outcomes..$^{23}$ However, these studies often focus on patientcentered communication during the medical interview and shared decision making. ${ }^{24,25}$ Results of this study describe the role of communication throughout a procedural treatment, or in this case, across medical acupuncture care. During medical acupuncture, this communication may carry even more power. A key step in performing acupuncture is patient feedback—de qi. Although not all medical procedures rely on patient feedback during the procedure, some techniques may be improved by eliciting patient information, such as therapeutic ultrasound, physical therapy, or osteopathic manipulation.

The final stage of the process is interlinked with health outcomes of acupuncture or its efficacy - an issue also addressed in phase one-which further highlights the importance of validating for patients that the treatment works. What also seems critical to address is that the health impact is not just physical, but also mental and social. From both physicians' and patients' perspectives, acupuncture is a treatment that appears to address health concerns, resulting in outcomes holistically, treating both body and mind. While previous research indicated that talking about outcomes is an important factor, these findings further illustrate that both physicians and patients identify complex, interrelated health outcomes that appear to be quite comprehensive (as opposed to just simply eliminating a physical ailment, like pain).

Future research can extend these findings to investigate the long-term impact of acupuncture, especially valuable within family medicine where we value continuity of care with our patients. The current results provide a model for physicians to integrate and talk though medical acupuncture with their patientsa model that warrants further testing as such training could improve a number of important outcomes (eg, enhancing patient engagement in acupuncture, physicians' self-effcacy, and patients' health outcomes).

Notable limitations to this study are reflective of the unique nature of the single site where interviews occurred. First, physicians in this hospital and residency are encouraged to complete medical acupuncture training, which has created a culture that embraces it as a treatment modality. Second, although researchers invited both patients who had and who had not reported successful treatment of acupuncture, we acknowledge that patients may be more motivated to share successful experiences. Given the exploratory nature of this initial study and the rarity of acupuncture treatment integrated into family medical settings, this is a first step in building knowledge in this realm of practice. Future research is needed to better understand the experience of patients who do not report notable results of acupuncture and to extend this study into family medicine settings that experience less "acupuncture saturation."

ACKNOWLEDGMENTS: The authors acknowledge Daniel Shaffer and Heather Rider for their support in recruitment and scheduling. Research support was also provided by the Military Primary Care Research Network.

This work was presented at the 2017 Society of Teachers of Family Medicine Annual Spring Conference in San Diego, CA, May 5-9, 2017.

FINANCIAL SUPPORT: This work was supported by the Acus Foundation.

DISCLAIMER: The opinions and assertions contained herein are the private views of the authors and are not to be construed as official or as reflecting the views of the US Air Force, the Uniformed Services University of the Health Sciences, the Department of Defense nor the US Government.

CORRESPONDING AUTHOR: Address correspondence to Dr Ledford, Uniformed Services University of the Health Sciences - Family Medicine, 4301 Jones Bridge Road, Bethesda, MD 20814. christian.ledford@usuhs.edu.

\section{References}

1. Gould A, MacPherson H. Patient perspectives on outcomes after treatment with acupuncture. J Altern Complement Med. 2001;7(3):261-268.

2. Coeytaux RR, Befus D. Role of acupuncture in the treatment or prevention of migraine, tension-type headache, or chronic headache disorders. Headache. 2016;56(7):1238-1240.

3. Moss DA, Crawford P. Ear acupuncture for acute sore throat: a randomized controlled trial. J Am Board Fam Med. 2015;28(6):697705 . 
4. Usichenko TI, Lehmann Ch, Ernst E. Auricular acupuncture for postoperative pain control: a systematic review of randomised clinical trials. Anaesthesia. 2008;63(12):1343-1348.

5. Helms JM. An overview of medical acupuncture. In: Jonas WB, Levin JS, eds. Essentials of Complementary and Alternative Medicine. Baltimore, MD: Williams \& Wilkins; 1999.

6. Sherman KJ, Cherkin DC, Eisenberg DM, Erro J, Hrbek A, Deyo RA. The practice of acupuncture: who are the providers and what do they do? Ann Fam Med. 2005;3(2):151-158.

7. Coeytaux RR, Park JJ. Acupuncture research in the era of comparative effectiveness research. Ann Intern Med. 2013;158(4):287-288.

8. Zhang Q, Yue J, Golianu B, Sun Z, Lu Y. Updated systematic review and meta-analysis of acupuncture for chronic knee pain. Acupunct Med. 2017;35(6):392-403.

9. Zhang Y, Lin L, Li H, Hu Y, Tian L. Effects of acupuncture on cancer-related fatigue: a metaanalysis. Support Care Cancer. 2018;26(2):415425.

10. Chou R, Deyo R, Friedly J, et al. Nonpharmacologic therapies for low back pain: a systematic review for an American College of Physicians clinical practice guideline. Ann Intern Med 2017;166(7):493-505

11. Murakami M, Fox L, Dijkers MP. Ear acupuncture for immediate pain relief-a systematic review and meta-analysis of randomized controlled trials. Pain Med. 2017;18(3):551-564.

12. Kaptchuk TJ, Kelley JM, Conboy LA, et al. Components of placebo effect: randomised controlled trial in patients with irritable bowel syndrome. BMJ. 2008;336(7651):999-1003.

13. Suarez-Almazor ME, Looney C, Liu Y, et al. A randomized controlled trial of acupuncture for osteoarthritis of the knee: effects of patientprovider communication. Arthritis Care Res (Hoboken). 2010;62(9):1229-1236.
14. Street RL Jr, Cox V, Kallen MA, Suarez-Almazor ME. Exploring communication pathways to better health: clinician communication of expectations for acupuncture effectiveness. Patient Educ Couns. 2012;89(2):245-251.

15. Yeh GY, Ryan MA, Phillips RS, Audette JF Doctor training and practice of acupuncture: results of a survey. J Eval Clin Pract. 2008;14(3):439-445.

16. Dillon PJ. Assessing the influence of patient participation in primary care medical interviews on recall of treatment recommendations. Health Commun. 2012;27(1):58-65.

17. Elwyn G, Frosch D, Thomson R, et al. Shared decision making: a model for clinical practice. J Gen Intern Med. 2012;27(10):1361-1367.

18. Elwyn G, Pickles T, Edwards A, et al. Supporting shared decision making using an Option Grid for osteoarthritis of the knee in an interface musculoskeletal clinic: A stepped wedge trial. Patient Educ Couns. 2016;99(4):571-577.

19. Glaser BG, Strauss AL. The Discovery of Grounded Research: Strategies for Qualitative Research. New York: Aldine De Gruyter; 1967

20. Strauss A, Corbin J. Basics of qualitative research: Techniques and procedures for developing grounded theory. Thousand Oaks, CA Sage; 1998

21. Corriere MA, Avise JA, Peterson LA, et al. Exploring patient involvement in decision making for vascular procedures. J Vasc Surg. 2015;62(4):1032-1039.e2.

22. Ehrich K, Cowie L, Sandall J. Expect the unexpected: patients' and families' expectations and experiences of new clinical procedures. Health Expect. 2015;18(5):918-928.

23. Stewart MA. Effective physician-patient communication and health outcomes: a review. CMAJ. 1995;152(9):1423-1433.
24. Epstein RM, Franks P, Fiscella K, et al. Measuring patient-centered communication in patient-physician consultations: theoretical and practical issues. Soc Sci Med. 2005;61(7):15161528.

25. Street RL. Communication in medical encounters: an ecological perspective. Handbook of health communication. Mahwah, NJ: Lawrence Erlbaum Associates; 2003. 